\title{
A loss-of-function variant in OSBPL1A predisposes to low plasma HDL cholesterol levels and impaired cholesterol efflux capacity
}

\section{Motazacker, Mahdi M.}

2016-06

Motazacker, M M , Pirhonen , J , van Capelleveen , J C , Weber-Boyvat , M , Kuivenhoven , J A , Shah , S , Hovingh , G K, Metso , J , Li , S , Ikonen, E, Jauhiainen , M , Dallinga-Thie , G M \& Olkkonen , V M 2016 , ' A loss-of-function variant in OSBPL1A predisposes to low plasma HDL cholesterol levels and impaired cholesterol efflux capacity ' , Atherosclerosis , vol. 249 , pp. 140-147 . https://doi.org/10.1016/j.atherosclerosis.2016.04.005

http://hdl.handle.net/10138/224029

https://doi.org/10.1016/j.atherosclerosis.2016.04.005

publishedVersion

Downloaded from Helda, University of Helsinki institutional repository.

This is an electronic reprint of the original article.

This reprint may differ from the original in pagination and typographic detail.

Please cite the original version. 


\title{
A loss-of-function variant in OSBPL1A predisposes to low plasma HDL cholesterol levels and impaired cholesterol efflux capacity
}

\author{
Mahdi M. Motazacker a, 2,1, Juho Pirhonen ${ }^{\text {b, } 2}$, Julian C. van Capelleveen ${ }^{\text {a }}$, \\ Marion Weber-Boyvat ${ }^{\text {c }}$, Jan Albert Kuivenhoven ${ }^{\text {d }}$, Saundarya Shah ${ }^{\text {c }}$, G. Kees Hovingh a , \\ Jari Metso ${ }^{\mathrm{e}}$, Shiqian Li ${ }^{\mathrm{b}}$, Elina Ikonen ${ }^{\mathrm{b}, \mathrm{c}}$, Matti Jauhiainen ${ }^{\mathrm{e}, 2}$, \\ Geesje M. Dallinga-Thie ${ }^{\mathrm{a}, \mathrm{f}, * *, 2}$, Vesa M. Olkkonen ${ }^{\mathrm{b}, \mathrm{c},{ }^{*}, 2}$
}

a Department of Vascular Medicine, Academic Medical Center (AMC), Amsterdam, The Netherlands

${ }^{\mathrm{b}}$ Department of Anatomy, Faculty of Medicine, University of Helsinki, Finland

${ }^{\mathrm{c}}$ Minerva Foundation Institute for Medical Research, Helsinki, Finland

d Department of Pediatrics, Section Molecular Genetics, University Medical Center Groningen, Groningen, The Netherlands

e National Institute for Health and Welfare, Genomics and Biomarkers Unit, Helsinki, Finland

${ }^{\mathrm{f}}$ Department of Experimental Vascular Medicine, AMC, Amsterdam, The Netherlands

\section{A R T I C L E I N F O}

\section{Article history:}

Received 28 January 2016

Received in revised form

2 April 2016

Accepted 5 April 2016

Available online 11 April 2016

\section{Keywords:}

Cholesterol efflux

High-density lipoprotein

Oxysterol-binding protein

OSBPL1A

Rare variant

\begin{abstract}
A B S T R A C T
Background and aims: Among subjects with high-density-lipoprotein cholesterol (HDL-C) below the 1st percentile in the general population, we identified a heterozygous variant OSBPL1A p.C39X encoding a short truncated protein fragment that co-segregated with low plasma HDL-C.

Methods: We investigated the composition and function of HDL from the carriers and non-carriers and studied the properties of the mutant protein in cultured hepatocytes.

Results: Plasma HDL-C and apolipoprotein (apo) A-I were lower in carriers versus non-carriers, whereas the other analyzed plasma components or HDL parameters did not differ. Sera of the carriers displayed a reduced capacity to act as cholesterol efflux acceptors $(p<0.01)$, whereas the cholesterol acceptor capacity of their isolated HDL was normal. Fibroblasts from a p.C39X carrier showed reduced cholesterol efflux to lipid-free apoA-I but not to mature HDL particles, suggesting a specific defect in ABCA1mediated efflux pathway. In hepatic cells, GFP-OSBPL1A partially co-localized in endosomes containing fluorescent apoA-I, suggesting that OSBPL1A may regulate the intracellular handling of apoA-I. The GFP-OSBPL1A-39X mutant protein remained in the cytosol and failed to interact with Rab7, which normally recruits OSBPL1A to late endosomes/lysosomes, suggesting that this mutation represents a lossof-function.

Conclusions: The present work represents the first characterization of a human OSBPL1A mutation. Our observations provide evidence that a familial loss-of-function mutation in OSBPL1A affects the first step of the reverse cholesterol transport process and associates with a low HDL-C phenotype. This suggests that rare mutations in OSBPL genes may contribute to dyslipidemias.
\end{abstract}

(C) 2016 Elsevier Ireland Ltd. All rights reserved.
Abbreviations: ABCA1, ATP-binding cassette transporter A-1; ER, Endoplasmic reticulum; GFP, Green fluorescent protein; GST, Glutathione-S-transferase; HDL-C, High-density-lipoprotein cholesterol; LE, Late endosomes; OSBP, Oxysterol-binding protein; OSBPL1A, Oxysterol-binding protein-like 1A; wt, Wild-type.

* Corresponding author. Minerva Foundation Institute for Medical Research, Biomedicum 2U, Tukholmankatu 8, FI-00290, Helsinki, Finland.

** Corresponding author. Department of Experimental Vascular Medicine, G1.142, AMC, Meibergdreef 9, 1105AZ Amsterdam, The Netherlands.

E-mail addresses: g.m.dallinga@amc.nl (G.M. Dallinga-Thie), vesa.olkkonen@ helsinki.fi (V.M. Olkkonen).

1 Current address: Laboratory of Genome Diagnostics, Department of Clinical Genetics, AMC, Amsterdam, The Netherlands.

2 These authors contribute equally to the work.

\section{Introduction}

Plasma high-density-lipoproteins (HDL) comprise a heterogeneous population of particles which differ in density, particle size, charge, and composition. Epidemiological studies have consistently shown an inverse correlation between plasma HDL cholesterol (HDL-C) as well as apolipoprotein A-I (apoA-I) levels and cardiovascular disease (CVD) risk [1,2]. However, recent failures of drugs that target HDL-C levels [3,4], together with evidence from Mendelian randomization studies indicate that HDL may not be causally 
related to cardiovascular disease [5,6]. A number of common and rare genetic variations have been shown to be associated with altered plasma HDL-C concentrations. The best recognized among these are cholesteryl ester transfer protein (CETP) and endothelial lipase (LIPG) loss-of-function variants which cause markedly raised HDL-C levels, but do not protect against coronary heart disease (CHD) [6-8]. Additional genetic variants associated with elevated HDL-C levels include TRIB1, APOA1/C3/A4/A5 gene cluster, ANGPTL4 and GALNT2 [6].

Loss-of-function mutants in APOAI, ABCA1 and LCAT cause extremely low HDL-C levels [9]. Whether these mutations cause increased CVD risk is still a matter of debate $[10,11]$. On the other hand, a hypothesis on the importance of HDL functionality has emerged, suggesting the presence of distinct functional HDL subpopulations $[12,13]$. In a previous study, we discovered an increased number of rare non-synonymous variants in lipid-related genes in a cohort of subjects with extreme plasma HDL-C concentrations $(<1$ st or $>99$ th percentile) [14]. Among the identified variants in subjects with low HDL-C $(<1 \%)$ there was a nonsense mutation p.C39X in OSBPL1A, which encodes a homolog of Oxysterol-binding protein, OSBP $[15,16]$.

The human OSBPL genes constitute a family with 12 members the protein products of which are characterized by a carboxyterminal OSBP-related ligand-binding domain (ORD). These proteins are suggested to act as sterol and phospholipid transporters or sensors at organelle interfaces [17,18]. OSBPL1A targets late endocytic compartments (LE) via the small GTPase Rab7 and the endoplasmic reticulum (ER) through VAMP-associated proteins (VAPA and -B) $[19,20]$. OSBPL1A regulates the interactions of LE with ER membranes in a sterol-specific manner. OSBPL1A controls the motility, subcellular distribution and tethering of the endosomes by bridging between the ER and LE and through regulation of dynein/ dynactin and homotypic fusion and protein sorting (HOPS) complexes [21-23].

The role of OSBPL1A in lipid metabolism remains to be elucidated. Overexpression of human OSBPL1A in mouse macrophages disturbed cholesterol efflux to HDL and enhanced atherogenesis in low-density lipoprotein receptor knock-out animals [24]. On the other hand, knock-down of Osbpl1a in mouse macrophages impaired the efflux of endocytosed LDL cholesterol to extracellular apoA-I, indicating that the function of Osbpl1a in LE may involve cross-talk with ATP-binding cassette transporter A1 (Abca1)mediated pathways of cholesterol efflux, and could thus impact the biogenesis of HDL in the liver and the intestine [22].

In the present study, we characterize the composition and function of HDL from heterozygous carriers of an OSBPL1A p.C39X mutation. We further demonstrate an abnormal subcellular localization and a functional defect of the mutant protein, suggesting that this OSBPL1A loss of function mutation affects cholesterol removal from cells associated with the low HDL-C phenotype in the mutant carriers.

\section{Materials and methods}

\subsection{Study subjects}

A cohort of individuals with very high $(\mathrm{n}=40)$ and very low $(\mathrm{n}=40)$ plasma HDL-C levels ( $<1$ st and $>99$ th percentile for age and gender) from the general population was studied to identify the genetic background underlying the HDL-C phenotype as described [14]. Coding sequence and exon-intron boundaries of 195 lipid-related genes and 78 lipid-unrelated genes were sequenced using Agilent Sureselect custom capture library on the Illumina HiSeq 2000 platform. In 1 individual with low HDL a nonsense mutation in OSBPL1A (NM_080597.2) was identified and further family expansion was done to evaluate co-segregation of each mutation with the low HDL-C phenotype. Genomic DNA was extracted from $10 \mathrm{ml}$ whole blood on an AutopureLS system (Gentra Systems, Minneapolis, MN) according to the manufacturer's protocol. The primers to amplify the coding and intron-exon boundaries were designed using Primer 3 software (exon 1 p.C39X; c.112-115dup: forward 5' - TCC AAT CTG TGG GGT TCT TC-3', reverse $5^{\prime}$ - CCC CTT TCT GTA TTA GCA GGT G-3' and sequenced as described elsewhere [25].

Written informed consent was obtained from all individuals and the study was approved by the Medical Ethical Committee of the Amsterdam Medical Center.

\subsection{Analytical procedures}

Blood was obtained after overnight fasting in EDTA-containing tubes and directly placed on ice. Plasma was isolated by centrifugation at $4{ }^{\circ} \mathrm{C}, 1700 \times \mathrm{g}$ for $15 \mathrm{~min}$ and stored at $-80^{\circ} \mathrm{C}$ for further analysis. Plasma and HDL phospholipids were analyzed using the Phospholipids B kit (Wako Chemicals, Osaka, Japan) or Pureauto S PL-kit (Daiichi Pure Chemicals, Tokyo, Japan); triglycerides using the Triglycerides GPO-PAP-kit (Roche Diagnostics, Mannheim, Germany); free cholesterol using the Wako Free Cholesterol C kit (Wako Chemicals) and total cholesterol using the Cholesterol CHOD-PAP kit (Roche Diagnostics). Human apolipoprotein E (apoE) was detected by ELISA as described previously with some modifications [26]. Quantitation of human apoA-I was performed with a sandwich ELISA. Briefly, the wells were coated with a polyclonal rabbit antibody against human apoA-I. The bound apoA-I protein was detected with a HRP-conjugated rabbit anti-human apoA-I immunoglobulin G (IgG) [26]. Protein concentration of isolated HDL fraction was determined using bovine serum albumin as standard [27].

\subsection{Isolation of high-density lipoprotein (HDL)}

HDL was isolated from serum samples by sequential ultracentrifugation using Beckman Optima TL Table-Top ultracentrifuge (Beckman Coulter, Brea, CA) and $\mathrm{KBr}$ for density adjustment [28]. Serum $(0.5 \mathrm{ml})$ was first adjusted to the density (d) of $1.019 \mathrm{~g} / \mathrm{ml}$ and the centrifuge tube filled with a $\mathrm{d}=1.019 \mathrm{~g} / \mathrm{ml} \mathrm{KBr}$ solution to the total volume of $3 \mathrm{ml}$. The samples were centrifuged at $5{ }^{\circ} \mathrm{C}$ for $2 \mathrm{~h}$ at $500,000 \times \mathrm{g}$. After centrifugation remnant particles as well as very low and intermediate density lipoproteins were recovered in the top $1 \mathrm{ml}$ fraction and the bottom was adjusted to a density of $1.063 \mathrm{~g} / \mathrm{ml}$ using solid $\mathrm{KBr}$, filled to $3 \mathrm{ml}$ with $\mathrm{d}=1.063 \mathrm{~g} / \mathrm{ml} \mathrm{KBr}$ solution and centrifuged again $\left(5^{\circ} \mathrm{C}, 3 \mathrm{~h}, 500,000 \times \mathrm{g}\right)$. The top $1 \mathrm{ml}$ fraction contained low-density lipoproteins (LDL). To isolate the HDL fraction, the LDL bottom fraction was adjusted with solid $\mathrm{KBr}$ to a density of $1.21 \mathrm{~g} / \mathrm{ml}$, the vials filled with $\mathrm{KBr} 1.21 \mathrm{~g} / \mathrm{ml}$ density solution and then centrifuged $\left(5^{\circ} \mathrm{C}, 18 \mathrm{~h}, 500,000 \times g\right)$. Total HDL was obtained in top $1 \mathrm{ml}$ fraction. The isolated HDL was dialyzed against phosphate-buffered saline (PBS, pH 7.4) and stored at $-80^{\circ} \mathrm{C}$ before analysis.

\subsection{Measurement of pre $\beta-H D L$ levels}

The ability of human plasma samples to generate pre $\beta$-HDL was analyzed by incubating plasma for $17 \mathrm{~h}$ at $37^{\circ} \mathrm{C}$ in the presence of a Lecithin-Cholesterol Acyltransferase (LCAT) inhibitor (1 $\mathrm{mM}$ iodoacetate). The formed pre $\beta$-HDL particles were quantified by resolving the post-incubation plasma samples with twodimensional crossed immunoelectrophoresis, as previously reported [29]. Briefly, the crossed immunoelectrophoresis consisted of an agarose electrophoresis in the first dimension for separation 
of lipoproteins with their pre $\beta, \beta$ and $\alpha$ mobility, followed by electrophoresis in the second dimension using agarose gel containing 7.5\% (v/v) rabbit anti-human apoA-I antiserum. For visualization, the gel was dried and stained with Coomassie brilliant blue R250 and the areas under the pre $\beta$-HDL and $\alpha$-HDL peaks were calculated. The pre $\beta$-HDL area was expressed as a percentage of the sum of $\alpha$-HDL and pre $\beta$-HDL areas. ApoA-I levels in the pre $\beta$-HDL and $\alpha$-HDL fractions were calculated using the total plasma apoA-I concentration.

\subsection{Measurement of serum CETP, LCAT, PLTP and PON-1 activities}

Serum cholesteryl ester transfer protein (CETP) activity was analyzed by a radiometric method as a transfer/exchange of radiolabeled $\left[{ }^{14} \mathrm{C}\right]$ cholesteryl oleate (Amersham Biosciences, Amersham, UK) between exogenously added human low-densitylipoprotein (LDL) and HDL, as reported [30]. Radioactivity in HDL as a measure of transfer activity was determined by liquid scintillation counting. The activity of CETP was expressed as $\mathrm{nmol} / \mathrm{ml} / \mathrm{h}$. The intra- and inter-assay CVs for CETP were 16 and 12\%. Phospholipid transfer protein (PLTP) activity $(\mu \mathrm{mol} / \mathrm{ml} / \mathrm{h})$ was determined with a radiometric method as described [31]. The intra- and inter-assay CVs for PLTP were 9 and 12\%. Lecithin-cholesterol acyltransferase (LCAT) activity was measured as described [32]. The interassay CV for LCAT was 4\%. Paraoxonase-1 (PON-1) activity was measured with a chromogenic method [33]. The intra-assay and inter-assay CVs for PON-1 measurements were 10 and 7\%, respectively.

\subsection{Cell culture}

Fibroblasts were obtained from participants by explant culture from a $3 \mathrm{~mm}$ punch at a $1 \mathrm{~mm}$ skin thickness. Primary cultures were grown in Dulbecco's Modified Eagle MEM (DMEM, Gibco/Thermo Fisher Scientific, Waltham, MA) supplemented with streptomycin $(100 \mu \mathrm{g} / \mathrm{ml})$ and penicillin $(100 \mathrm{U} / \mathrm{ml})$ and $20 \%$ fetal calf serum (FCS) and maintained at $37^{\circ} \mathrm{C}$ and $5 \% \mathrm{CO}_{2}$ in stock flasks. Cells were used till passage 15. Mouse Raw264.7 macrophages were cultured in DMEM (Sigma-Aldrich, St. Louis, MO), 10 mM HEPES, pH 7.4, 10\% FCS (Gibco), $100 \mathrm{U} / \mathrm{ml}$ penicillin, and $100 \mu \mathrm{g} / \mathrm{ml}$ streptomycin, and the human HuH7 hepatoma cell line in Eagle's minimal essential medium with Earle's salts (EMEM, Sigma-Aldrich) with the above supplements.

\subsection{Cholesterol efflux from fibroblasts}

For the efflux experiments fibroblasts cells were cultured in 24wells until confluency [34]. Cells were loaded with cholesterol including $0.5 \mu \mathrm{Ci} / \mathrm{ml}\left[{ }^{3} \mathrm{H}\right]$ cholesterol as a tracer in labeling medium (DMEM:F12 Glutamax supplemented with $0.1 \% \mathrm{p} / \mathrm{s}, 0.2 \%$ BSA, $30 \mu \mathrm{g} / \mathrm{ml}$ cholesterol) for $24 \mathrm{~h}$ in the presence or absence of $3 \mu \mathrm{M}$ LXR agonist T0901317 (Cayman Chemicals, Ann Arbor, MI). After labeling efflux was initiated by adding $20 \mu \mathrm{g} / \mathrm{ml}$ human HDL (Calbiochem, San Diego, CA) or $10 \mu \mathrm{g} / \mathrm{ml}$ lipid-free apoA-I (Calbiochem) for $4 \mathrm{~h}$, after which the efflux medium was removed. Cells were extracted with 2-propanol. The presence of $\left[{ }^{3} \mathrm{H}\right]$ cholesterol in both medium and cell extracts was assessed in a liquid scintillation counter (TriCarb 2900TR, Packard, London, UK). Fractional cholesterol efflux equals the counts in medium divided by the counts in the medium + cell extract, subtracted by the counts in medium without HDL or apoA-I.

\subsection{Cholesterol efflux from Raw264.7 macrophages}

Raw264.7 macrophages seeded on 24-well plates were loaded for $48 \mathrm{~h}$ with $50 \mu \mathrm{g} / \mathrm{ml}$ (as total protein) acetylated LDL labeled with $\left[{ }^{3} \mathrm{H}\right]$ cholesterol oleate (Amersham Biosciences, Amersham, UK) and prepared in a medium containing 5\% lipoprotein-deficient serum [29]. After washes, cholesterol efflux was carried out for $16 \mathrm{~h}$ to serum-free medium containing either $1 \%$ serum or $25 \mu \mathrm{g} / \mathrm{ml}$ (as total protein) purified HDL from the subjects under investigation. The radioactivity in the efflux media and in the cells was thereafter determined by liquid scintillation counting, and efflux \% calculated.

\section{9. cDNA constructs}

The p.C39X mutation was introduced into OSBPL1A cDNA (AF323726) in pEGFP-C1 (Clontech/TaKaRa Bio, Mountain View, CA) with the QuikChange kit (Agilent, Santa Clara, CA). The wild-type and p.C39X mutant constructs, as well as the plain pEGFP-C1 vector, were used for transfection experiments. For recombinant protein production OSBPL1A CDNA fragments encoding aa 1-208 and 1-38 were generated by PCR and cloned into pGEX1 $\lambda T$ (GE Healthcare, Little Chalfont, UK).

\subsection{Fluorescence microscopy}

HuH7 hepatoma cells were transfected with pEGFP-C1-OSBPL1A-wt or -39X expression constructs using Lipofectamine 2000 (Invitrogen/Life Technologies, Carlsbad, CA). After 24 h, Alexa568dextran $(100 \mu \mathrm{g} / \mathrm{ml}$; Molecular Probes/Life Technologies, Eugene, OR) was added for $90 \mathrm{~min}$ in growth medium to label endocytic compartments. Alternatively, $20 \mu \mathrm{g} / \mathrm{ml}$ of lipid-free human apoA-I (kindly donated by Dr. Peter Lerch, the Swiss Red Cross) labeled with Alexa Fluor 568 protein labeling kit (Thermo Fisher, Waltham, MA) was added in serum-free DMEM, $1 \mathrm{mg} / \mathrm{ml}$ fatty acid-free BSA, and incubated for $90 \mathrm{~min}$ at $37{ }^{\circ} \mathrm{C}$. The cells were fixed and mounted for fluorescence microscopy. In some cases LE/lysosomes were stained with anti-Lamp1 H4A3 (Santa Cruz Biotechnology, Dallas, TX). The specimens were imaged using a Leica (Wetzlar, Germany) SP8 TCS confocal microscope and a $63 \times$ HC PL APO CS2 water immersion objective. Image stacks were deconvoluted using WPL-method (Wiener Filter Preconditioned Landweber) in Parallel Iterative Deconvolution plugin for Fiji.

For co-localization analysis, the image stacks were transformed into sum projection images and non-specific background was removed. Mander's co-localization coefficient was quantified from manually segmented cells using Fiji. Student's $t$-test was used to assess statistical difference of means.

\subsection{Co-immunoprecipitation of OSBPL1A with Rab7}

To assess the interaction of OSBPL1A-39X with Rab7, HuH7 cells were cotransfected with OSBPL1A-wt or -39X in pEGFP-C1 (Clontech/TaKaRa Bio, Mountain View, CA) or the empty vector, and Xpress $^{\text {TM }}$-Rab7 cDNA in pcDNAHisMaxC. After washes, the cells were lysed in $10 \mathrm{mM}$ Hepes, $\mathrm{pH} 7.4,150 \mathrm{mM} \mathrm{NaCl}, 0.5 \mathrm{mM} \mathrm{MgCl}_{2}$, 10\% glycerol, 1 mM DTT, 0.5\% Triton X-100, EDTA-free protease inhibitor cocktail (Roche Diagnostics, Basel, Switzerland) and centrifuged to remove cell debris. Cleared lysates were incubated overnight at $+4{ }^{\circ} \mathrm{C}$ with anti-Xpress ${ }^{\mathrm{TM}}$ monoclonal antibody (Invitrogen/Life Technologies) and immune complexes were pulled down with protein G Sepharose 4 Fast Flow (GE Healthcare). Precipitates were analyzed by SDS-PAGE and Western blotting using antibodies against the Xpress ${ }^{\mathrm{TM}}$ epitope and anti-GFP (A6455, Life Technologies).

\subsection{Pull-down with glutathione-S-transferase fusion proteins}

GST fusion proteins were produced in E. coli BL21 (DE3) by 
A

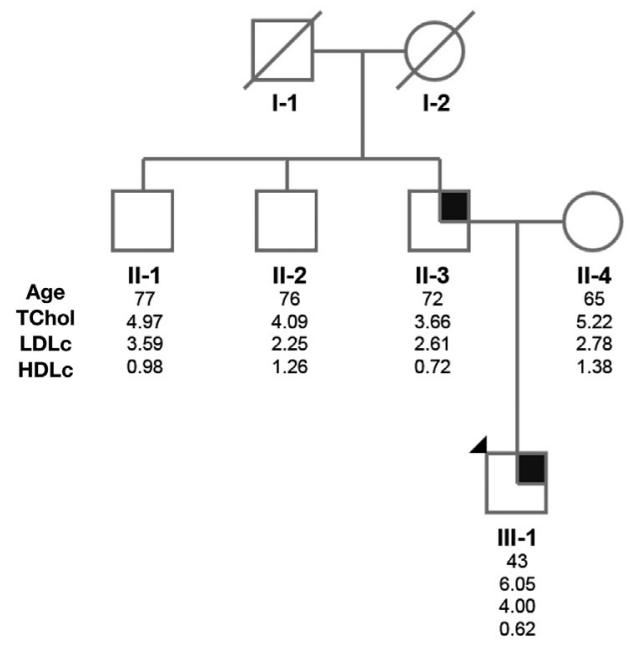

B

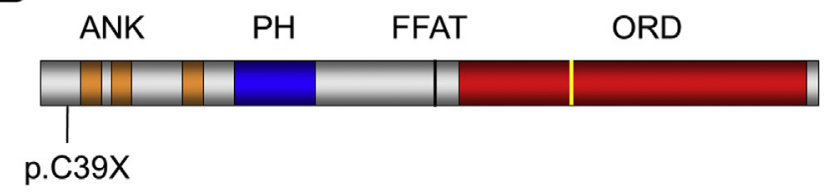

C

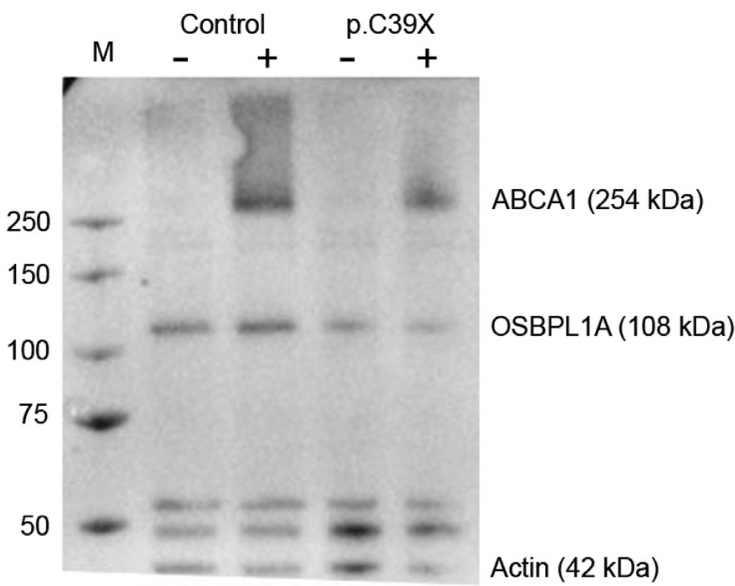

Fig. 1. Co-segregation of the low plasma HDL cholesterol with the heterozygous OSBPL1A p.C39X c.112-115dup mutation. (A) The pedigree characterized with a heterozygous nonsense mutation in OSBPL1A represented by a black square is shown. Fo each individual age, plasma total cholesterol, LDL-C and HDL-C (all in mmol/L) are depicted. (B) Schematic presentation of the OSBPL1A protein structure with functional domains and the mutation site indicated. ANK, ankyrin repeats; $\mathrm{PH}$, pleckstrin homology domain; FFAT, two phenylalanines in an acidic tract; ORD, OSBP-related ligandbinding domain. (C) Western blot analysis of total protein extracts from fibroblasts isolated from a p.C39X carrier and a non-carrier family member. Specimens treated for $24 \mathrm{~h}$ with the LXR agonist T0901317 $(3 \mu \mathrm{M})$ are indicated with $(+)$. Top: ABCA1 induced by LXR; Middle: OSBPL1A; Bottom: $\beta$-actin (loading control). Mobilities of molecular mass markers are indicated on the left.

employing a standard protocol. HuH7 cells transfected for $24 \mathrm{~h}$ with Xpress $^{\mathrm{TM}}$-Rab7 were lysed in $20 \mathrm{mM}$ Hepes, pH 7.4, $100 \mathrm{mM} \mathrm{K}$ acetate, $5 \mathrm{mM} \mathrm{MgCl}_{2}, 1 \mathrm{mM}$ DTT, 0.5\% Triton X-100, EDTA-free protease inhibitor cocktail (Roche Diagnostics, Mannheim, Germany) and subjected to pull-down with $10 \mu \mathrm{g}$ GST or GST fusion proteins and Glutathione-Sepharose 4B (GE Healthcare). After washes with the above buffer without Triton X-100 and the
Table 1

Characteristics of the non-carriers and carriers of the OSBPL1A-p.C39X mutation.

\begin{tabular}{|c|c|c|c|c|}
\hline & \multicolumn{2}{|c|}{ Non-carriers } & \multicolumn{2}{|c|}{ Carriers } \\
\hline & II-4 & II-1 & III-1 & II-3 \\
\hline Gender (F/M) & $\mathrm{F}$ & M & M & M \\
\hline Age (yrs) & 65 & 77 & 43 & 72 \\
\hline BMI $\left(\mathrm{kg} / \mathrm{m}^{2}\right)$ & 27 & 31 & 27 & 27 \\
\hline Cholesterol (mmol/L) & 5.22 & 4.97 & 6.05 & 3.66 \\
\hline $\mathrm{LDL}-\mathrm{C}(\mathrm{mmol} / \mathrm{L})$ & 2.78 & 3.59 & 4.00 & 2.61 \\
\hline HDL-C (mmol/L) & 1.38 & 0.98 & 0.62 & 0.72 \\
\hline apoA-I (g/L) & 1.63 & 0.84 & 0.74 & 0.65 \\
\hline apoE g/L & 31.4 & 49.7 & 34.2 & 34.8 \\
\hline PLTP $\mu \mathrm{mol} / \mathrm{ml} / \mathrm{h}$ & 5.31 & 7.10 & 6.64 & 6.92 \\
\hline CETP nmol $/ \mathrm{ml} / \mathrm{h}$ & 33.4 & 40.2 & 24.9 & 17.0 \\
\hline LCAT $\mathrm{nmol} / \mathrm{ml} / \mathrm{h}$ & 29.6 & 30.4 & 23.0 & 30.7 \\
\hline $\mathrm{PON}-1 \mu \mathrm{mol} / \mathrm{min}$ & 10.5 & 15.4 & 8.7 & 11.4 \\
\hline
\end{tabular}

${ }^{\text {a }}$ Individual II-2 (Fig. 1A) was not willing to participate in the study.

protease inhibitors, the beads were boiled with Laemmli sample buffer, separated on 10\% SDS-PAGE, and Western blotted with antiXpress $^{\mathrm{TM}}$.

\subsection{Bioinformatics}

For analysis of the OSBPL1A p.C39X mutation the European ancestries in the Exome Variant Server database (ESP, http://evs.gs. washington.edu/EVS/) and the Genome of the Netherlands database (Go-NL, http://www.rug.nl/research/genetics/databases/ genomeofthenetherlands/) [35,36] were used.

\subsection{Statistical analyses}

Because of the small number of individuals included in the study we provide individual data. The non-parametric Kruskal-Wallis test or Student's $t$-test were performed to test for significant differences. The statistical results were calculated with SPSS vs22 software (IBM, Armonk, NY).

\section{Results}

\subsection{Gene and protein analysis of OSBPL1A p.C39X variant}

The heterozygous genetic variant p.C39X (c.112-115dup) in OSBPL1A gene was identified in a recent population screen to detect the underlying genetic abnormality in patients with extremely low plasma HDL-C levels below the 1st percentile [14](Fig. 1A). The 4nucleotide duplication causes a frameshift that replaces cysteine 39 with a stop codon. The allele thus encodes a short, truncated

Table 2

HDL composition and pre- $\beta$-HDL proportions in the OSBPL1A p.C39X non-carriers and carriers.

\begin{tabular}{|c|c|c|c|c|}
\hline \multirow{2}{*}{$\begin{array}{l}\text { Genotype } \\
\text { Subject }\end{array}$} & \multicolumn{2}{|c|}{ Non-carrier } & \multicolumn{2}{|c|}{ Carrier } \\
\hline & II-4 & II-1 & III-1 & II-3 \\
\hline${ }^{\mathrm{a}}$ Pre- $\beta$-HDL \% & 8 & 10 & 8 & 8 \\
\hline${ }^{\mathrm{b}}$ Pre- $\beta$-HDL \% & 30 & 49 & 30 & 30 \\
\hline${ }^{\mathrm{C}}$ Cholesterol mass \% & 23 & 26 & 25 & 19 \\
\hline Phospholipids mass \% & 39 & 26 & 42 & 39 \\
\hline Triglycerides mass \% & 4 & 11 & 8 & 13 \\
\hline apoA-I mass \% & 34 & 37 & 25 & 29 \\
\hline apoE $\mu \mathrm{g} / \mathrm{ml}$ & 7.5 & 4.3 & 4.3 & 3.7 \\
\hline
\end{tabular}

a Pre- $\beta$-HDL proportion, starting value.

b Pre- $\beta$-HDL proportion after $17-\mathrm{h}$ incubation at $+37^{\circ} \mathrm{C}$. The pre $\beta$-HDL level is expressed as a percentage of the sum of $\alpha$-HDL and pre $\beta$-HDL.

c HDL mass \% composition was calculated based on cholesterol, phospholipid, triglyceride and apoA-I concentrations in isolated HDL. 

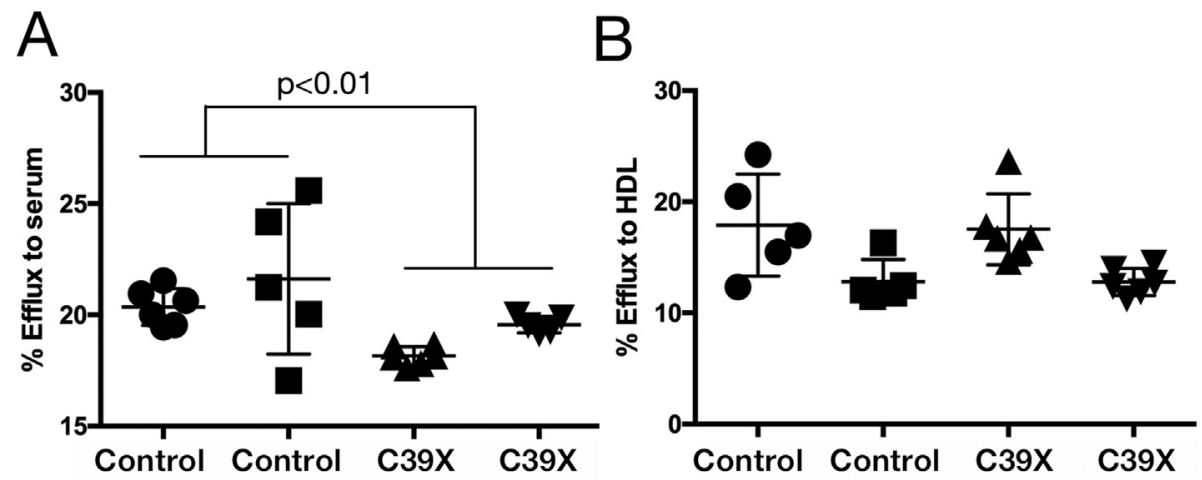

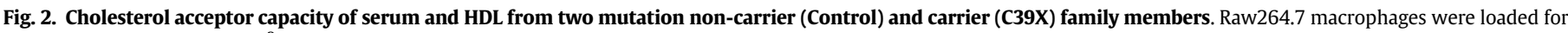

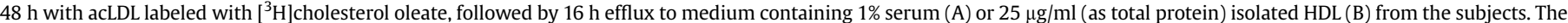

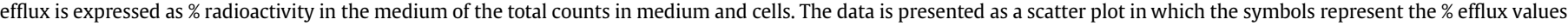

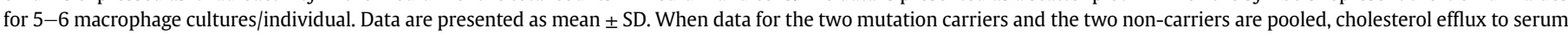

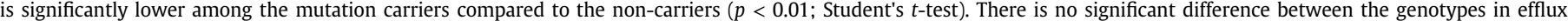
capacity to isolated HDL.
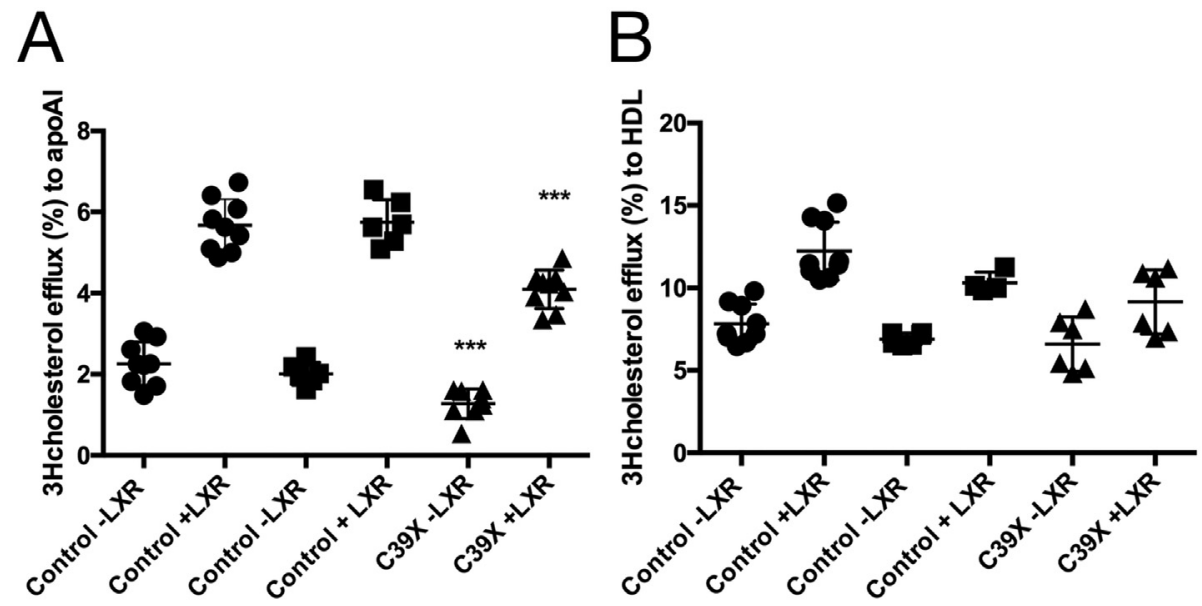

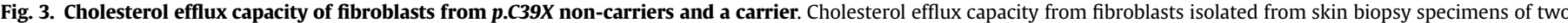

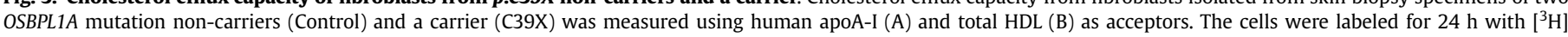

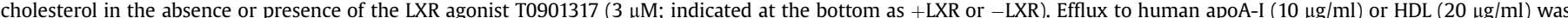

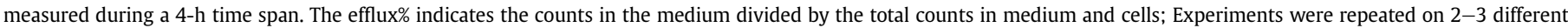
days, in triplicate on each day (each symbol represents an individual measurement). Statistical significance was tested using Student's $t$-test; ${ }^{* * *} p<0.001$ versus each control.

amino-terminal protein fragment. The p.C39X variant was found with a frequency of $0.38 \%$ in European ancestries in the Exome Variant Server database (ESP), however, no mutation carriers were found in The Genome of the Netherlands database (Go-NL) [35,36]. The mutation was not found in 40 patients with elevated plasma HDL-C levels above the 95th percentile. The site of the mutation in OSBPL1A protein is depicted in Fig. 1B. Western blot analysis of fibroblasts derived from a $p . C 39 X$ carrier revealed a reduced cellular level of full-length OSBPL1A protein (Fig. 1C), consistent with the notion that the variant allele carriers have a reduced dose of functional OSBPL1A protein in their isolated fibroblasts. In contrast to the response in ATP-binding cassette transporter A-1 (ABCA1) western blotted for a comparison, the cellular level of OSBPL1A protein could not be induced with the liver $X$ receptor (LXR) agonist T0901317 in either non-carriers or carriers of the mutation, suggesting that OSBPL1A is not a target of LXRs.

\subsection{Biochemical analysis of plasma}

Two carriers and two non-carriers of the OSBPL1A mutation were identified in the family, and the mutation co-segregated with the low HDL-C phenotype (Fig. 1A). The plasma HDL-C and apoA-I concentrations in the mutation carriers were markedly lower as compared to non-carriers (Fig. 1A, Table 1). None of the other lipid parameters nor lipid transfer proteins, LCAT or paraoxonase-1 (PON-1) activities were different among carriers and non-carriers of the OSBPL1A variant (Table 1). Analysis of the HDL composition and pre- $\beta$-HDL level and its formation capacity revealed no significant differences between the carriers and non-carriers (Table 2).

\subsection{The OSBPL1A p.C39X variant and cholesterol efflux}

We tested the capacity of the serum and HDL derived from the two heterozygous OSBPL1A p.C39X mutation carriers and two noncarrier relatives to act as acceptors in a macrophage cholesterol efflux assay, the first step in the reverse cholesterol transport process. We found that cholesterol efflux capacity from $\left[{ }^{3} \mathrm{H}\right]$ cholesterol-acLDL-loaded Raw264.7 macrophages to serum from the carriers was significantly $(p<0.01)$ reduced as compared to non-carriers (Fig. 2). When cholesterol efflux to purified HDL of the carriers at an equal protein concentration $(25 \mu \mathrm{g} / \mathrm{ml})$ was assessed, no consistent difference was observed. 

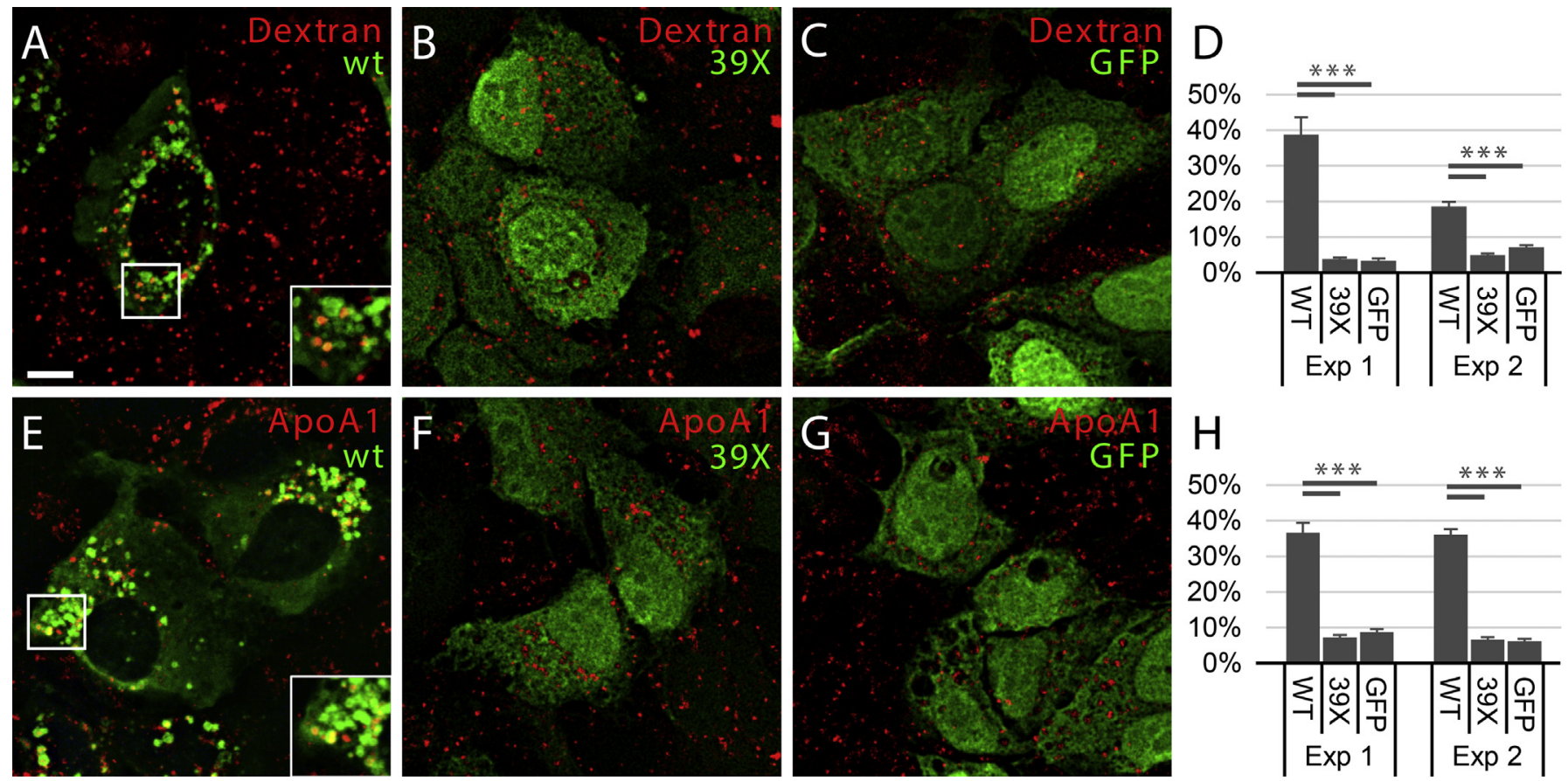

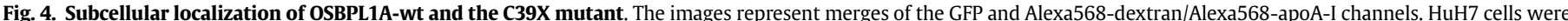

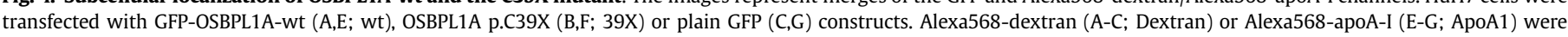

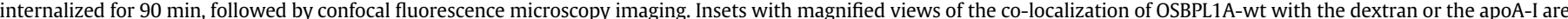

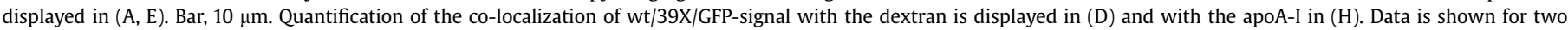
independent experiments (Exp1, Exp2); ${ }^{* * *} p<0.001$.

To study the effect of the OSBPL1A mutation on cellular cholesterol efflux capacity, fibroblasts derived from a p.C39X carrier and two non-carrier controls were tested. $\left[{ }^{3} \mathrm{H}\right]$ cholesterol efflux to human HDL or lipid-free apoA-I was measured in the absence or presence of an LXR agonist. The fibroblasts derived from the p.C39X carrier showed under both conditions significantly reduced cholesterol efflux to purified lipid-free apoA-I (Fig. 3A; $p<0.001$ ) but not to mature HDL particles (Fig. 3B), as compared to the controls.

\subsection{The OSBPL1A p.C39X variant encodes a soluble protein fragment}

GFP fusions of OSBPL1A-wt and the -39X variant were overexpressed in $\mathrm{HuH} 7$ cells to monitor their subcellular targeting. In accordance with Johansson et al. [19], wild-type (wt) GFP-OSBPL1A localized on clustered or fused large perinuclear late endosomal (LE) organelles, many of which co-localized with the late endosome/lysosome marker Lamp1 (data not shown) and with the internalized fluid-phase endocytic marker Alexa568-dextran (Fig. 4A). Moreover, the protein co-localized in some endosomes with Alexa568-labeled apoA-I internalized into the cells (Fig 4E). The apoA-I-containing organelles were occasionally encircled by the OSBPL1A signal, demonstrating that they represent apoA-I internalized in endosomes decorated by OSBPL1A or by ER cisternae with associated OSBPL1A [37]. OSBPL1A-39X, the short truncated $\mathrm{N}$-terminal fragment, was only present in the cytosolic compartment and in the nucleus (Fig. 4B,F), and was practically indistinguishable from the GFP control (Fig. 4C,G). Quantification of the fluorescence signals and their overlap revealed a significant colocalization of GFP-OSBPL1A-wt, but not the C39X fragment, with both internalized markers (Fig. 4D,H).

OSBPL1A is known to be recruited onto LE/lysosomes by the small GTPase Rab7, which interacts with the N-terminal region of
OSBPL1A containing three ankyrin repeats $[19,20]$. We therefore employed a co-immunoprecipitation approach to study whether the N-terminal OSBPL1A-39X might have the capacity to interact with Rab7. Cells were transfected with GFP, GFP-OSBPL1A-wt or GFP-OSBPL1A-p.39X, together with Xpress ${ }^{\text {TM }}$-Rab7, followed by immunoprecipitation with anti-Xpress ${ }^{\mathrm{TM}}$ and Western blot analysis of the precipitates. While OSBPL1A-wt precipitated abundantly with Rab7, none of the OSBPL1A-39X fusion protein was found in the precipitate (Fig. 5), strongly suggesting that the $39 \mathrm{X}$ fragment fails to bind Rab7. This result was confirmed by a pull-down assay employing lysates of cells transfected with Xpress ${ }^{\mathrm{TM}}$-Rab7 and a purified GST-39X fusion protein: While the positive control, OSBPL1A N-terminal fragment (aa 1-208), pulled-down Rab7, the GST39X failed to do so (Fig. 5B).

\section{Discussion}

In a previous study we identified a variant in the OSBPL1A gene, p.C39X, in subjects with very low ( $<1$ st percentile) plasma HDL-C levels [14]. The p.C39X variant results in a truncated protein consisting of only $38 \mathrm{~N}$-terminal amino acid residues of OSBPL1A. We now demonstrate that this variant represents a loss-of-function leading to a reduced dose of functional OSBPL1A protein in fibroblasts of the carriers.

Plasma HDL-C and apoA-I levels were reduced in heterozygous carriers of the OSBPL1A mutation, and plasma from the carriers showed a decreased cholesterol efflux acceptor capacity from cholesterol-loaded macrophages, consistent with the reduced concentration of HDL-C and apoA-I. Concomitantly, isolated HDL from the mutation carriers, when employed at equal protein concentration as acceptor, displayed no defect in cholesterol efflux, suggesting that the properties of endogenous HDL in this functional assay are not severely affected. Moreover, fibroblasts from an OSBPL1A p.C39X mutation carrier were defective in cholesterol 


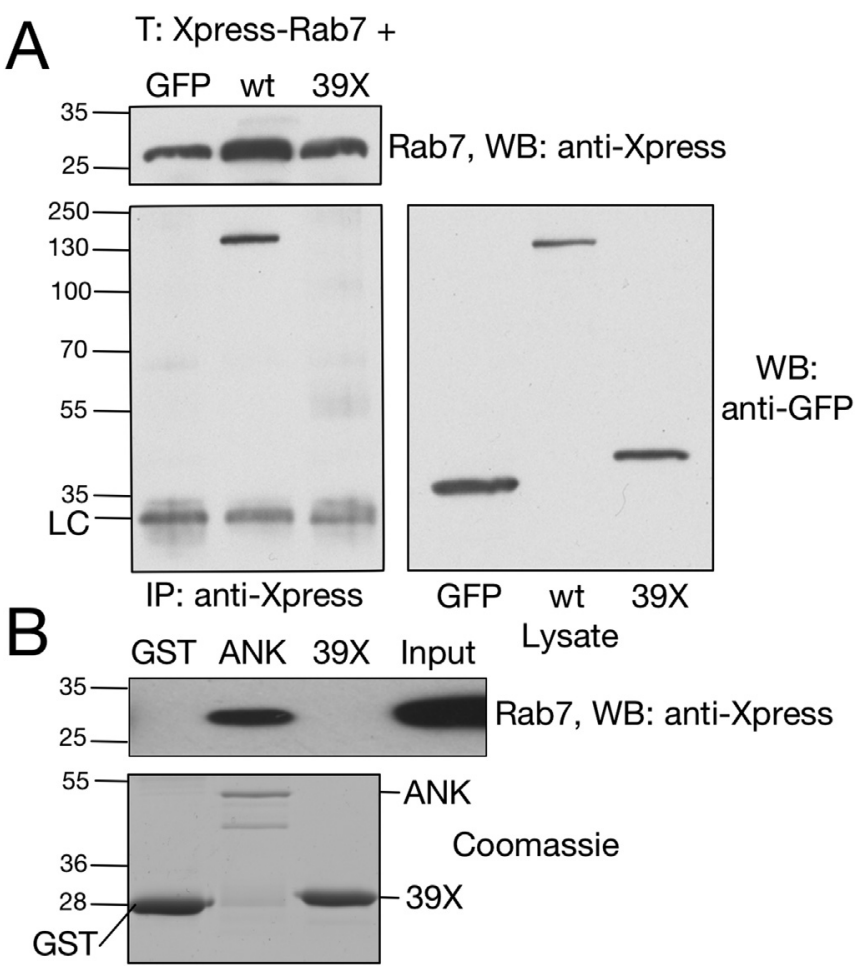

Fig. 5. OSBPL1A-39X does not interact with Rab7. (A) HuH7 cells cotransfected (T) with Xpress ${ }^{\mathrm{TM}}$-Rab7 and an empty pEGFP vector (GFP), GFP-OSBPL1A-wt (wt), or the p.C39X mutant (identified on the top) were subjected to immunoprecipitation (IP) with Xpress $^{\mathrm{TM}}$ antibody. The precipitates were Western blotted with anti-Xpress ${ }^{\mathrm{TM}}$ and antiGFP (indicated on the right). Mobilities of molecular mass markers are displayed on the left; LC, immunoglobulin light chain. A blot of cell lysates demonstrating expression of control GFP and the fusion proteins in shown on the right. (B) Lysates of cells transfected with Xpress ${ }^{\mathrm{TM}}$-Rab7 were subjected to pull-down with purified GST, GSTOSBPL1A (1-208) encoding the N-terminal fragment (aa 1-208) with ankyrin repeats (ANK), or GST-39X (39X), followed by Western blotting with Xpress ${ }^{\mathrm{TM}}$ antibody. The 'Input' lane contains $1 / 25$ of the input lysate. The fusion proteins used ( $2.5 \mu \mathrm{g}$ total protein/lane) are depicted in the Coomassie-stained bottom panel.

efflux to lipid-free apoA-I but not to mature HDL particles, indicating a specific defect in ABCA1-mediated efflux.

OSBPL1A has previously been connected with the cellular cholesterol homeostasis as illustrated in conditions of overexpression as well as knock-down of the protein. Defective cholesterol efflux from cholesterol-loaded macrophages to HDL particles was observed in transgenic mice overexpressing human OSBPL1A under scavenger receptor A promoter [24]. On the other hand, Vihervaara et al. [22] observed that knock-down of Osbpl1A with RNAi in Raw264.7 macrophages resulted in defective cholesterol efflux capacity to apoA-I, which is similar to the phenotype observed in our OSBPL1A mutant. The function of OSBPL1A has thus far not been studied in tissues responsible for bulk HDL biogenesis, the liver and the intestine [38]. However, the reported functional links with cholesterol efflux to apoA-I or HDL indicate that OSBPL1A, which is expressed in both liver [39] and intestine (http:// biogps.org/), is likely to contribute to the biogenesis and/or secretion of HDL particles [40]. In the present experiments we showed that fluorescent apoA-I added in the medium of cultured hepatocytes transfected with OSBPL1A partially co-localized with the OSBPL1A in endosomal compartments. This observation is consistent with the notion that OSBPL1A may regulate the intracellular trafficking and handling of apoA-I.

The short amino-terminal OSBPL1A-39X fragment was localized in the cytosolic compartment, while the wild-type OSBPL1A localizes to late endocytic compartments via an interaction with the small GTPase Rab7 [19,20] and regulates late endosome/lysosome motility, tethering and fusion $[20,22,23]$. The present data demonstrate that the OSBPL1A-39X fragment is unable to interact with Rab7, consistent with the lack of LE/lysosomal localization. This fragment also lacks the region between the pleckstrin homology domain and the ORD that mediates the homodimerization of OSBP [41,42], heterodimerization of OSBP with OSBP2/ORP4L [42], and ORP9 with ORP11 [43]. This strongly suggests that the 39X fragment is unable to dimerize with full-length OSBPL1A encoded by the other, intact allele in the heterozygous variant carriers, and to perturb its function in a dominant fashion. The above observations provide evidence that the OSBPL1A-39X protein fragment represents a loss-of-function. Whether this loss-of-function affects the secretion of apoA-I from the liver or intestine remains to be investigated.

Until now, we have only identified one nuclear family with a nonsense mutation in OSBPL1A. Sequencing of more subjects with the low HDL-C phenotype will hopefully lead to the identification of additional OSBPL1A mutation carriers to allow replication of the present findings and survey of more detailed mechanisms behind the low HDL-C. Further study is required to unequivocally establish whether the OSBPL1A p.C39X mutation has a causal relationship with the low-HDL phenotype; At present we cannot exclude the possibility that unknown linked mutation(s) may have an impact on the observed low-HDL phenotype.

To conclude, our data suggest that the $p . C 39 X$ loss-of-function mutation identified in OSBPL1A affects cholesterol removal from cells and associates with the low plasma HDL cholesterol levels. The present work represents the first report on a human OSBPL1A mutation and raises the possibility that rare mutations in OSBPL genes may contribute to dyslipidemias.

\section{Conflict of interest}

The authors declared that they do not have anything to disclose regarding conflict of interest with respect to this manuscript.

\section{Financial support}

GKH is holder of a Veni grant [91612122] from the Netherlands Organisation for Scientific Research (NWO). This work is supported by CardioVascular Research Initiative [CVON2011-19; Genius], the European Union [Resolve: FP7-305707 and TransCard: FP7603091-2] and the Academy of Finland grants No. 257545 (M.J.), 285223 (V.M.O.), and 272130 and 282192 (E.I.). Further support was obtained from the Sigrid Jusélius Foundation (V.M.O, E.I.), the Finnish Foundation for Cardiovascular Research (V.M.O, E.I.), the Finnish Diabetes Research Foundation, the Liv och Hälsa Foundation, and the Magnus Ehrnrooth Foundation (V.M.O.).

\section{Acknowledgements}

We would like to acknowledge all participants. We thank K. Los for the genetic fieldwork and A.W.M. Schimmel, J. Peter, T. Willems, E. Jääskeläinen, R. Kosonen, and U. Tienhaara for technical and laboratory assistance.

\section{References}

[1] T. Gordon, W.P. Castelli, M.C. Hjortland, W.B. Kannel, T.R. Dawber, High density lipoprotein as a protective factor against coronary heart disease. The Framingham study, Am. J. Med. 62 (1977) 707-714.

[2] W.E. Boden, High-density lipoprotein cholesterol as an independent risk factor in cardiovascular disease: assessing the data from Framingham to the veterans affairs high-density lipoprotein intervention trial, Am. J. Cardiol. 86 (2000) 19L-22L. 
[3] P.J. Barter, M. Caulfield, M. Eriksson, S.M. Grundy, J.J. Kastelein, M. Komajda, J. Lopez-Sendon, L. Mosca, J.C. Tardif, D.D. Waters, C.L. Shear, J.H. Revkin, K.A. Buhr, M.R. Fisher, A.R. Tall, B. Brewer, Illuminate Investigators, Effects of torcetrapib in patients at high risk for coronary events, N. Engl. J. Med. 357 (2007) 2109-2122.

[4] G.G. Schwartz, A.G. Olsson, M. Abt, C.M. Ballantyne, P.J. Barter, J. Brumm, B.R. Chaitman, I.M. Holme, D. Kallend, L.A. Leiter, E. Leitersdorf, J.J. McMurray, H. Mundl, S.J. Nicholls, P.K. Shah, J.C. Tardif, R.S. Wright, DAL out come investigators, Effects of dalcetrapib in patients with a recent acute coronary syndrome, N. Engl. J. Med. 367 (2012) 2089-2099.

[5] C.L. Haase, A. Tybjaerg-Hansen, A.A. Qayyum, J. Schou, B.G. Nordestgaard, R.L.C.A.T. Frikke-Schmidt, HDL cholesterol and ischemic cardiovascular disease: a mendelian randomization study of HDL cholesterol in 54,500 individuals, J. Clin. Endocrinol. Metab. 97 (2012) E248-E256.

[6] B.F. Voight, G.M. Peloso, M. Orho-Melander, R. Frikke-Schmidt, M. Barbalic, M.K. Jensen, et al., Plasma HDL cholesterol and risk of myocardial infarction: a mendelian randomisation study, Lancet 380 (2012) 572-580.

[7] A.B. Baker, A. Groothuis, M. Jonas, D.S. Ettenson, T. Shazly, E. Zcharia, I. Vlodavsky, P. Seifert, E.R. Edelman, Heparanase alters arterial structure, mechanics, and repair following endovascular stenting in mice, Circ. Res. 104 (2009) 380-387.

[8] D.J. Rader, G.K. Hovingh, HDL and cardiovascular disease, Lancet 384 (2014) 618-625.

[9] D.J. Rader, E.M. deGoma, Approach to the patient with extremely low HDLcholesterol, J. Clin. Endocrinol. Metab. 97 (2012) 3399-3407.

[10] F. Oldoni, R.J. Sinke, J.A. Kuivenhoven, Mendelian disorders of high-density lipoprotein metabolism, Circ. Res. 114 (2014) 124-142.

[11] A.E. Bochem, D.F. van Wijk, A.G. Holleboom, R. Duivenvoorden, M.M. Motazacker, G.M. Dallinga-Thie, E. de Groot, J.J. Kastelein A.J. Nederveen, G.K. Hovingh, E.S. Stroes, ABCA1 mutation carriers with low high-density lipoprotein cholesterol are characterized by a larger atherosclerotic burden, Eur. Heart J. 34 (2013) 286-291.

[12] D.J. Rader, A.R. Tall, The not-so-simple HDL story: is it time to revise the HDL cholesterol hypothesis? Nat. Med. 18 (2012) 1344-1346.

[13] D. Saleheen, R. Scott, S. Javad, W. Zhao, A. Rodrigues, A. Picataggi, D. Lukmanova, M.L. Mucksavage, R. Luben, J. Billheimer, J.J. Kastelein, S.M. Boekholdt, K.T. Khaw, N. Wareham, D.J. Rader, Association of HDL cholesterol efflux capacity with incident coronary heart disease events: a prospective case-control study, Lancet Diabetes Endocrinol. 3 (2015) 507-513.

[14] M.M. Motazacker, J. Peter, M. Treskes, C.C. Shoulders, J.A. Kuivenhoven, G.K. Hovingh, Evidence of a polygenic origin of extreme high-density lipoprotein cholesterol levels, Arterioscler. Thromb. Vasc. Biol. 33 (2013) $1521-1528$.

[15] M. Lehto, S. Laitinen, G. Chinetti, M. Johansson, C. Ehnholm, B. Staels, E. Ikonen, V.M. Olkkonen, The OSBP-related protein family in humans, J. Lipid Res. 42 (2001) 1203-1213.

[16] C.J. Jaworski, E. Moreira, A. Li, R. Lee, I.R. Rodriguez, A family of 12 human genes containing oxysterol-binding domains, Genomics 78 (2001) 185-196.

[17] V.M. Olkkonen, S. Li, Oxysterol-binding proteins: sterol and phosphoinositide sensors coordinating transport, signaling and metabolism, Prog. Lipid Res. 52 (2013) 529-538.

[18] J. Moser von Filseck, B. Mesmin, J. Bigay, B. Antonny, G. Drin, Building lipid 'PIPelines' throughout the cell by ORP/Osh proteins, Biochem. Soc. Trans. 42 (2014) 1465-1470.

[19] M. Johansson, M. Lehto, K. Tanhuanpää, T.L. Cover, V.M. Olkkonen, The oxysterol-binding protein homologue ORP1L interacts with Rab7 and alters functional properties of late endocytic compartments, Mol. Biol. Cell 16 (2005) $5480-5492$.

[20] M. Johansson, N. Rocha, W. Zwart, I. Jordens, L. Janssen, C. Kuijl, V.M. Olkkonen, J. Neefjes, Activation of endosomal dynein motors by stepwise assembly of Rab7-RILP-p150Glued, ORP1L, and the receptor betalll spectrin, J. Cell Biol. 176 (2007) 459-471.

[21] N. Rocha, C. Kuijl, R. van der Kant, L. Janssen, D. Houben, H. Janssen, W. Zwart, J. Neefjes, Cholesterol sensor ORP1L contacts the ER protein VAP to control Rab7-RILP-p150 Glued and late endosome positioning, J. Cell Biol. 185 (2009) 1209-1225.

[22] T. Vihervaara, R.L. Uronen, G. Wohlfahrt, I. Björkhem, E. Ikonen, V.M. Olkkonen, Sterol binding by OSBP-related protein $1 \mathrm{~L}$ regulates late endosome motility and function, Cell Mol. Life Sci. 68 (2011) 537-551.

[23] R. van der Kant, A. Fish, L. Janssen, H. Janssen, S. Krom, N. Ho,
T. Brummelkamp, J. Carette, N. Rocha, J. Neefjes, Late endosomal transport and tethering are coupled processes controlled by RILP and the cholesterol sensor ORP1L, J. Cell Sci. 126 (2013) 3462-3474.

[24] D. Yan, M. Jauhiainen, R.B. Hildebrand, K. Willems van Dijk, T.J. Van Berkel, C. Ehnholm, M. Van Eck, V.M. Olkkonen, Expression of human OSBP-related protein $1 \mathrm{~L}$ in macrophages enhances atherosclerotic lesion development in LDL receptor-deficient mice, Arterioscler. Thromb. Vasc. Biol. 27 (2007) 1618-1624.

[25] C. Candini, A.W. Schimmel, J. Peter, A.E. Bochem, A.G. Holleboom, M. Vergeer, R.P. Dullaart, G.M. Dallinga-Thie, G.K. Hovingh, K.L. Khoo, T. Fasano, L. Bocchi, S. Calandra, J.A. Kuivenhoven, M.M. Motazacker, Identification and characterization of novel loss of function mutations in ATP-binding cassette transporter A1 in patients with low plasma high-density lipoprotein cholesterol, Atherosclerosis 213 (2010) 492-498.

[26] S. Siggins, M. Jauhiainen, V.M. Olkkonen, J. Tenhunen, C. Ehnholm, PLTP secreted by HepG2 cells resembles the high-activity PLTP form in human plasma, J. Lipid Res. 44 (2003) 1698-1704.

[27] O.H. Lowry, N.J. Rosebrough, A.L. Farr, R.J. Randall, Protein measurement with the Folin phenol reagent, J. Biol. Chem. 193 (1951) 265-275.

[28] R.J. Havel, H.A. Eder, J.H. Bragdon, The distribution and chemical composition of ultracentrifugally separated lipoproteins in human serum, J. Clin. Investig. 34 (1955) 1345-1353.

[29] R. Vikstedt, J. Metso, J. Hakala, V.M. Olkkonen, C. Ehnholm, M. Jauhiainen, Cholesterol efflux from macrophage foam cells is enhanced by active phospholipid transfer protein through generation of two types of acceptor particles, Biochemistry 46 (2007) 11979-11986.

[30] J.E. Groener, R.W. Pelton, G.M. Kostner, Improved estimation of cholesteryl ester transfer/exchange activity in serum or plasma, Clin. Chem. 32 (1986) $283-286$.

[31] M. Jauhiainen, C. Ehnholm, Determination of human plasma phospholipid transfer protein mass and activity, Methods 36 (2005) 97-101.

[32] M. Jauhiainen, P.J. Dolphin, Human plasma lecithin-cholesterol acyltransferase. An elucidation of the catalytic mechanism, J. Biol. Chem. 261 (1986) 7032-7043.

[33] P. Kleemola, R. Freese, M. Jauhiainen, R. Pahlman, G. Alfthan, M. Mutanen, Dietary determinants of serum paraoxonase activity in healthy humans, Atherosclerosis 160 (2002) 425-432.

[34] R. Franssen, A.W. Schimmel, S.I. van Leuven, S.C. Wolfkamp, E.S. Stroes, G.M. Dallinga-Thie, In vivo inflammation does not impair ABCA1-mediated cholesterol efflux capacity of HDL, Cholesterol 2012 (2012) 610741.

[35] L.C. Francioli, P.P. Polak, A. Koren, A. Menelaou, S. Chun, I. Renkens, Genome of the Netherlands C, C.M. van Duijn, M. Swertz, C. Wijmenga, G. van Ommen, P.E. Slagboom, D.I. Boomsma, K. Ye, V. Guryev, P.F. Arndt, W.P. Kloosterman, P.I. de Bakker, S.R. Sunyaev, Genome-wide patterns and properties of de novo mutations in humans, Nat. Genet. 47 (2015) 822-826.

[36] E.M. van Leeuwen, L.C. Karssen, J. Deelen, A. Isaacs, C. Medina-Gomez, H. Mbarek, et al., Genome of The Netherlands population-specific imputations identify an ABCA6 variant associated with cholesterol levels, Nat. Commun. 6 (2015) 6065

[37] M. Weber-Boyvat, H. Kentala, J. Peränen, V.M. Olkkonen, Ligand-dependent localization and function of ORP-VAP complexes at membrane contact sites, Cell Mol. Life Sci. 72 (2015) 1967-1987.

[38] V.I. Zannis, P. Fotakis, G. Koukos, D. Kardassis, C. Ehnholm, M. Jauhiainen, A. Chroni, HDL biogenesis, remodeling, and catabolism, Handb. Exp. Pharmacol. 224 (2015) 53-111.

[39] M. Johansson, V. Bocher, M. Lehto, G. Chinetti, E. Kuismanen, C. Ehnholm, B. Staels, V.M. Olkkonen, The two variants of oxysterol binding protein-related protein-1 display different tissue expression patterns, have different intracellular localization, and are functionally distinct, Mol. Biol. Cell 14 (2003) 903-915.

[40] M.C. Phillips, Molecular mechanisms of cellular cholesterol efflux, J. Biol. Chem 289 (2014) 24020-24029.

[41] N.D. Ridgway, P.A. Dawson, Y.K. Ho, M.S. Brown, J.L. Goldstein, Translocation of oxysterol binding protein to Golgi apparatus triggered by ligand binding, J. Cell Biol. 116 (1992) 307-319.

[42] J.P. Wyles, R.J. Perry, N.D. Ridgway, Characterization of the sterol-binding domain of oxysterol-binding protein (OSBP)-related protein 4 reveals a novel role in vimentin organization, Exp. Cell Res. 313 (2007) 1426-1437.

[43] Y. Zhou, S. Li, M.I. Mäyränpää, W. Zhong, N. Bäck, D. Yan, V.M. Olkkonen, OSBP-related protein 11 (ORP11) dimerizes with ORP9 and localizes at the Golgi-late endosome interface, Exp. Cell Res. 316 (2010) 3304-3316. 